\title{
An Exhaustive Survey on Meta-Heuristic Algorithms for Unimodal and Multimodal Optimization
}

\author{
K. Kalaivani, N. UmaMaheswari
}

\begin{abstract}
A review of the global feature color comprising of seven different metrics are discussed here. The color is the most powerful feature for describing the images. This study contains four sections. The first section explains the related techniques used in various papers. The second section explains the two different kinds of metrics. (1) Similarity metrics such as Cosine and Correlation and (2) Dissimilarity metrics such as Euclidean, Manhattan, Bhattacharyya, Chi-Squared and Intersection. The third section explains experiment results using CALTECHUCSD Birds-200 image library. The fourth section gives the conclusion and future work. In this experiment, the query image can be divided into trained (indexed) or untrained (non-indexed). In the similarity metric analysis, the experimental results show that the cosine similarity gives better similarity score than correlation. Similarly, in the dissimilarity metric analysis, the Bhattacharyya gives a better result than other distance metrics.
\end{abstract}

Key Words: Color histogram, Similarity metric, Dissimilarity metric, Cosine similarity, Correlation Euclidean, Manhattan, Bhattacharyya, Chi-Squared and Intersection

\section{INTRODUCTION}

Today latest optimization technique problems which include NP-hard problems are involved with exponential computation time complexity for most Meta heuristic problems. These exponential computation times are needed to solve in worst case scenario. We find some optimal solution from any feasible solutions. This kind of Meta heuristic problems could be solved in a valid when compared with other solutions because of its computation time. These Meta heuristic problems do not take excessive time to find solution for a particular problem.

Heuristic Algorithms such as Genetic and PSO use stochastic search to find Optimum solutions. In first iterations they attempt to intensification (recognition) new regions and find local optimums. In final iteration they try to increase the accuracy of search and diversification (exploration) in order to converge to global optimums. Adjusting intensification and diversification has important role in optimization with Heuristic Algorithms [1]. Finding the regions with standard quality solutions also do not spend much time in the regions.[2].

In this proposed work namely firefly, bat and Chicken Swarm search algorithms are compared and are experimented. The Bat algorithm was elucidated by XinSheYang in 2010.The X.-S.Yang was began to grow

Revised Manuscript Received on January 2, 2020.

K. Kalaivani, PSNA College of Engineering \& Technology, Department of Computer Science \& Engineering, Dindigul,Tamil Nadu, India.

Dr. N. UmaMaheswari, PSNA College of Engineering \& Technology, Department of Computer Science \& Engineering, Dindigul,Tamil Nadu, India
Firefly Algorithm (FA) in 2007. The Chicken Swarm was expounded by X.-S.Yang and S.Deb in 2009. With the help of experimental results, the comparison between the three algorithms namely, Bat algorithm (BA), Firefly Algorithm (FA) and Chicken Swarm Search (CS) Algorithm for Unimodal and Multimodal Optimization are carried out. We finally conclude this paper that the Chicken Swarm search algorithm is better when compared with other two algorithms.

\section{ALGORITHM \& OPTIMIZATION}

\section{A. Firefly Algoritm (Ffa)}

Firefly is the one which live in humid environment also belong to insect family.These fireflies yield cold light chemically and their color varies as yellow, green, pale-red light. These colors are created on the flashing patterns and performance of fireflies.

Performance and nature of Fireflies

Fireflies are the beings that can yield light enclosed by it. Light creation in fireflies is owing to a kind of chemical reaction [3]. The main persistence for firefly's flash is to turn as a signal system to draw the care of other fireflies. Although they have many mechanisms, the captivating issues are communication between them in order to locate the food also they protect themselves from enemies such as hunters and to guard themselves from adversary hunters, challenging reproduction and so on.Nearly there are three thousand firefly species are considered [4].These fireflies make tiny and rhythmic flashes. Fireflies which create these rhythmic flashes are unique species. A unique pattern is designed through the tempo of flashing and amount of time taken to form. These unique patterns are fascinated by both male and female fireflies. The similar species of male and female retort to individual pattern. The light intensity at a definite distance from the light source trails the inverse square law. That is due to the distance rises the light intensity decreases [2].

Moreover, the air absorbs light which becomes weaker and weaker as there is arise of the distance. There are two issues collectively involved in firefly. The first oneis the most fireflies perceptible only to a partial distance that is frequently good enough to interconnect each other. The flashing light can be articulated in such a way that it is associated with the objective function to be optimized. This makes it possible to express new metaheuristic algorithms [3]. 
The fireflyalgorithmis a metaheuristic algorithm, stimulated by the flashing activities of fireflies [1]. The most vitalfortitude of a firefly's flash is to try as a signal system to enticementcare of other fireflies [3]. Xin-She Yang stated this algorithm using three norms. All fireflies are unisexual, so that one firefly will be fascinated to all other fireflies. Charisma is relational to their luminosity, and for any two fireflies, the less bright one will be paying attention to the brighter one [4]; however, the brightness can diminish as their distance increases. If there are no fireflies brighter than a given firefly, it will move indiscriminately [4].

Light Intensity and Attractiveness

Firefly algorithm have some of the issues, they are light intensity that vary, attractiveness that are formulated [2]. There are various demerits appear in firefly algorithm. In firefly the brightness I for a particular location $\mathrm{x}$ is chosen as $\mathrm{I}(\mathrm{x}) \propto \mathrm{f}(\mathrm{x})$ to maximize the optimization problems. The attractiveness $\beta$ is judged by the other fireflies [4]. Therefore, it could be able to distinguish the distance rijamong firefly $\mathrm{i}$ and firefly $\mathrm{j}$. In totting up, from source the light intensity becomes light and it isabsorbed, so it could allow the attractiveness to vary with the varying degree of absorption [1].

\section{B. Bat Algorithm}

Bat-inspired algorithms or Bat algorithms could be developed with the help of some of the echolocation characteristics of microbats [5]. The following idealized rules are given below:

a) In order to sense distance all bats use the echolocation and all bat sense the difference between food or prey and background in some way.

b) Bats search for prey and they randomly glide with a static frequency fmin, by changeable wavelength $\lambda[4]$.

c) The loudness varies from $\mathrm{A} 0$ to a minimum constant value Amin positively.

The moral feature for the application in computational geometry is tracing is not used to estimate the time delay[5]. Let the range of wavelengths $[\lambda \mathrm{min}, \lambda \max ]$ be $0.8 \mathrm{~mm}$ to $18 \mathrm{~mm}$ and the frequency $\mathrm{f}$ in a range [fmin, fmax] could be $[30 \mathrm{kHz}, 550 \mathrm{kHz}]$ considered.In order to make the implementation so simple any wavelength could be used. In the actual implementation, it could adjust the range by adjusting the frequencies, and the detectable range [6].

The wavelength $\lambda$ and $\mathrm{f}$ are related such that $\lambda \mathrm{f}$ is constant. In general, the fact is that the frequency travel in a short distance who have higher frequencies with short wavelengths [8]. For bats, the distinctive ranges are a few meters [7]. In Bat the range of pulse could be 0 which means no pulses to 1 means the extreme rate of pulse emission.

Using the following formulas, the virtual bats which are used naturally in simulation, define new solution for their position and velocity in a d-dimensional search space [8].

$$
\begin{gathered}
f_{i}=f_{\min }+\left(f_{\max }-f_{\min }\right) \beta(1) \\
v_{i}^{t}=v^{t-1}{ }_{i}+\left(x_{i}^{t}-x_{*}\right) f_{i}(2) \\
x_{i}^{t}=x^{t-1}{ }_{i}+v^{t}{ }_{i}(3)
\end{gathered}
$$

Through a uniform distribution a random vector is drawn [5]. Here $\beta$ belongs to 0 or 1 and $x^{*}$ is the best solution when compared with all $n$ bats solutions. It could use either fi or $\lambda \mathrm{i}$ as velocity which could adjust its velocity while fixing the other factor $\lambda \mathrm{i}[6]$.

\section{Chicken Swarm Search Algorithm (Csa)}

Chicken Swarm is a bird known for their aggressive reproductive strategy [14]. Chicken Swarm birds are meant for breeding system. In order to raise the young one Chicken Swarm brood parasitism either with the same species or with different species. By laying their eggs on other's nest they improve their survival probability of their own. Also, they destroy or remove other's eggs from that particular nest. Due to this some may involve in direct conflict with the interfering Chicken Swarms. Chicken Swarms built new nest if they are defeated. [14]. They reduce the probability of their eggs being abandoned by laying their eggs which are identified closely resemble the eggs of their host.

Chicken Swarms choose the nest where the host ones laid their eggs recently. The Chicken Swarm egg hatch their young one earlier when compared with the host ones. Also, the Chicken Swarm's chick grows faster than the host birds. The egg present in the nest indicate solution. The egg laid by Chicken Swarm represent new solution [12]. The inferior solution could be replaced by creating a new and possibly better solution using Chicken Swarm. Normally each nest is constructed with one egg. The new algorithm helps to create multiple eggs in a nest which represent a set of solutions. [11]. The Chicken Swarm search algorithm could be stated such that Chicken Swarm lay an egg and produce a set of solution by laying it randomly in a nest. By laying the egg randomly in various nest it could produce various solutions with best eggs which helps to carry over to next generation. Due to fixed number of nest the host bird has the probability of discovering the egg or discard the egg. If it discards the egg or nest then new nest could be constructed in new location. Lévy Flight is performed by generating various new solution for ithChicken Swarm egg. [11].

Chicken Swarm functions are tested with various optimization algorithms. The optimization algorithm which are involved in this comparison are tested and their results are analyzed. The three bat, firefly and Chicken Swarm are validated using seven test functions and also, they are validated. Ackley is one of the function used to test the above-mentioned algorithms.

The range of global minimum could be $(x *)=0$ at $x *=(0$, $0 \ldots, 0) x i \in[-32.768,32.768]$, for all $i=1,2, \ldots, \mathrm{d}$.

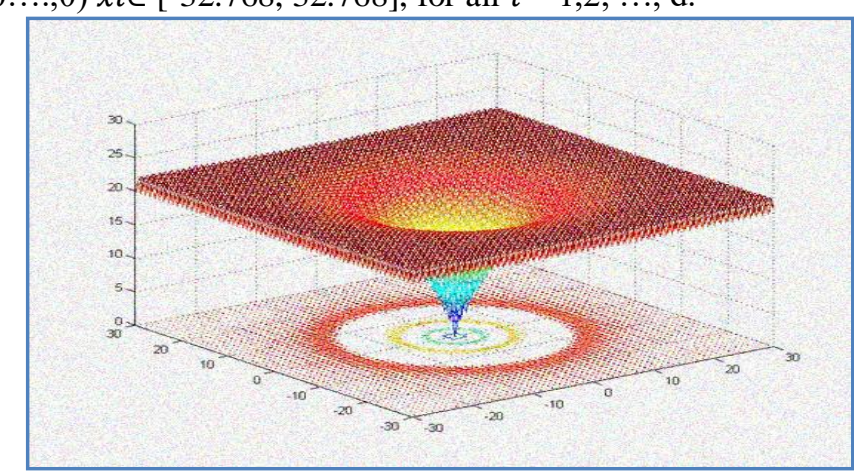

Fig. 1 Testing Ackley Optimization Function 
Egg holder function is a challenging function to optimize, for the reason that of the enormousnumber of local minima.Egg holder function hold minimum function value which is identified as a multimodal test function $f(x *)$ $=-959.6407$, at $x *=(512,404.2319)$ in range $x i \in[-512$, 512], for all $\mathrm{i}=1,2$.

The Styblinski-Tang function is regularly evaluated on the hypercube xi $\in[-5,5]$, for all $i=1, \ldots, d$. TheStyblinskiTang function is given below by its equations and shown here in its two-dimensional form

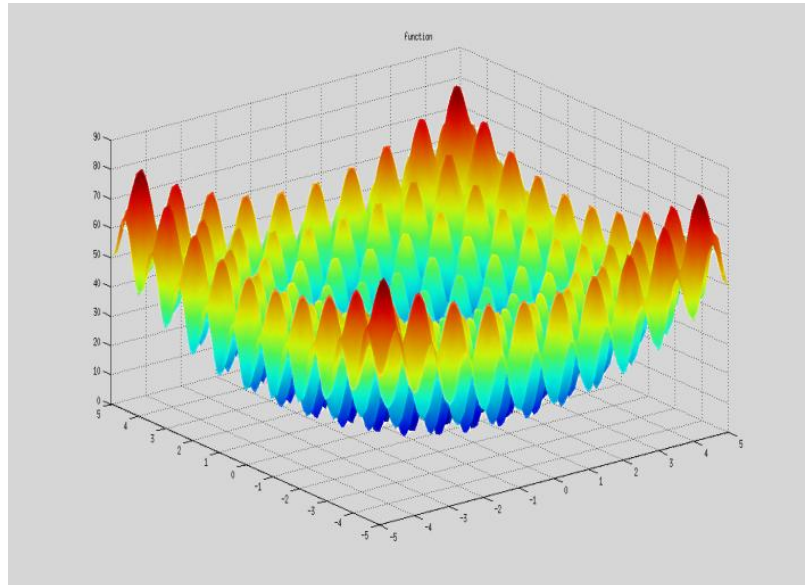

Fig. 2 Testing Egg Holder Optimization Function

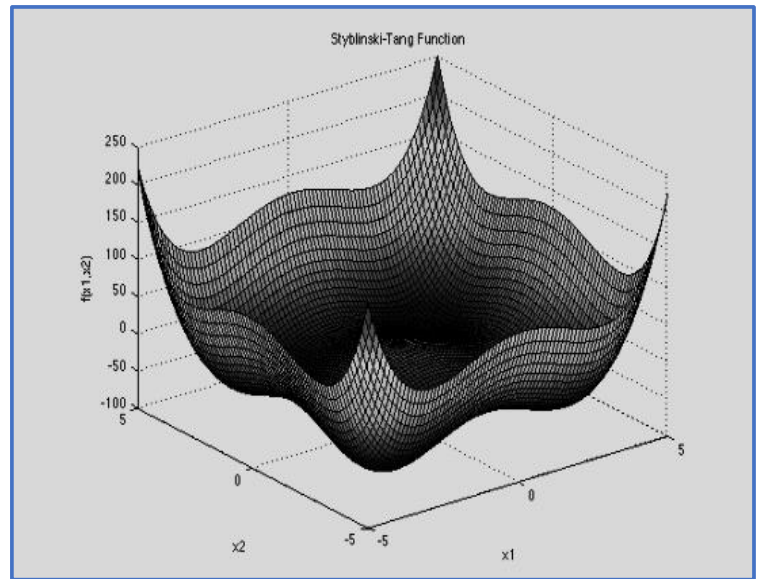

Fig.3 Testing Styblinski-Tang Optimization Function

The fourth Schaffer function is revealed on a smaller input domain in the second plot to show point. The fourth Schaffer function is frequently evaluated on the square xi $\in$ $[-100,100]$, for all $\mathrm{i}=1$

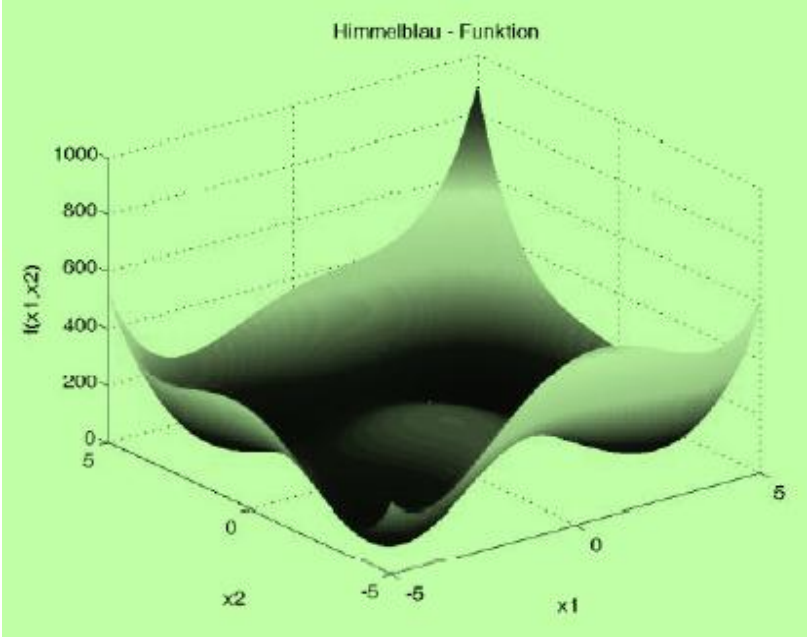

Fig. 4 Testing Fourth Schaffer Optimization Function
The Mccormick function is generally estimated on the rectangle $\mathrm{x} 1 \in[-1.5,4], \mathrm{x} 2 \in[-3,4]$.

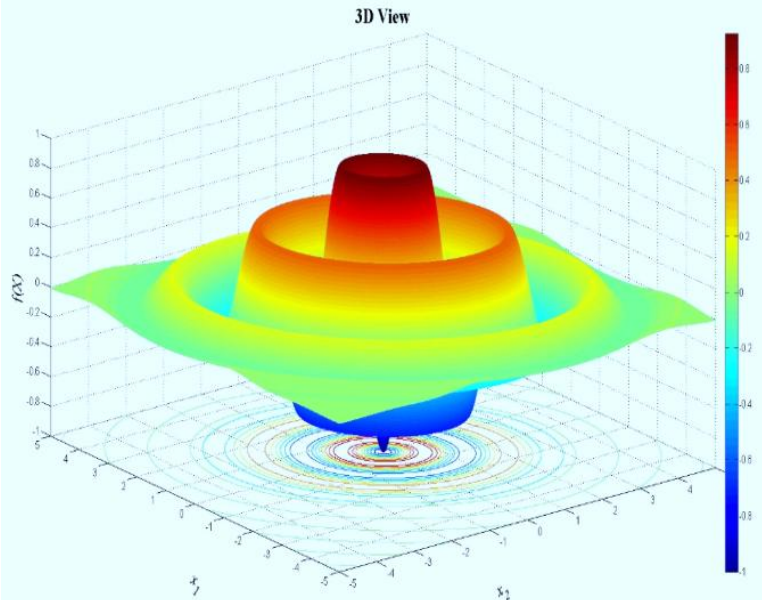

\section{Fig 5. Testing Mccormick Function Optimization} Function

The Himmelblau's function is named after David MautnerHimmelblau. In mathematical optimization, Himmelblau's function is a multi-modal function, used to examine the presentation of optimization algorithms. The function could be defined through the locations of all the least can be found analytically. However, because they are roots of cubic polynomials, when written in terms of radicals, the expressions are somewhat complicated.

The three-hump Camel function on its suggested input domain, and the plot displays only a portion of this domain, to permit for easier viewing of the function's key characteristics. The three-hump Camel function has three local minima. The three-hump Camel function is typically estimated on the square $x i \in[-5,5]$, for all $i=1,2$.

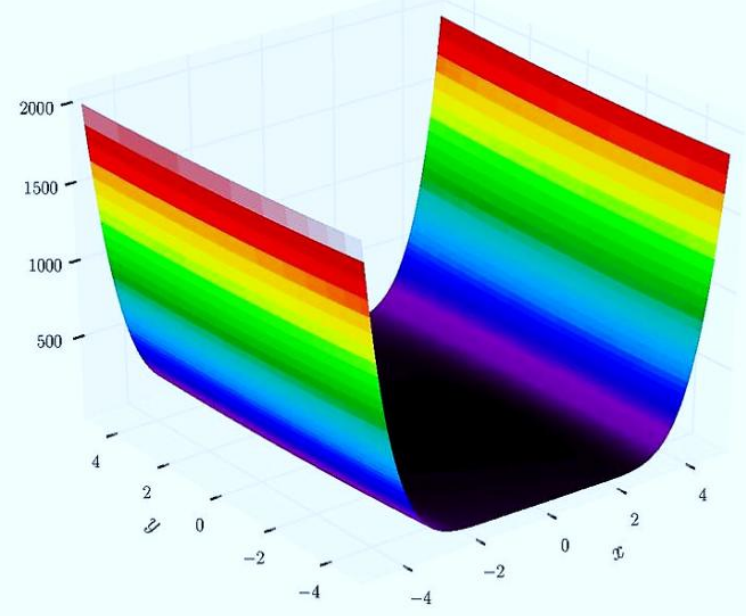

Fig 6. Testing Himmelblau's Function Optimization Function

\section{I.Optimization For Unimodal Test Function}

In this part the three algorithm Firefly, Bat and Chicken Swarm are compared using seven unimodal test functions for optimization. Unimodal test functions are easier to optimize. They have only single local minima. 


\section{A. Fitness Calculation}

All unimodal test functions which is mentioned above are used and we have calculated the mean fitness value using them. Table 1 explains the Comparison of Optimization Fitness for Unimodal Test Functions which is given below.

Table 1Optimization Fitness Comparison for Unimodal Test Functions

\begin{tabular}{|c|l|c|c|c|}
\hline S.NO & FUNCTIONS & BAT & FFA & CS \\
\hline 1 & Ackley & 0.5975 & 0.5668 & 0.5667 \\
\hline 2 & Egg holder & 21.89 & 4.49 & $1.63 \mathrm{e}-14$ \\
\hline 3 & Styblinski-Tang & 4.973 & $2.19 \mathrm{e}-06$ & $1.77 \mathrm{e}-28$ \\
\hline 4 & Fourth Schaffer & 0.0199 & 0.001169 & $4.27 \mathrm{e}-09$ \\
\hline 5 & The Mccormick & $8.34 \mathrm{e}+2$ & $0.52 \mathrm{e}+03$ & $0.52 \mathrm{e}+03$ \\
\hline 6 & Himmelblau's & $\begin{array}{c}1.92 \mathrm{e}- \\
11\end{array}$ & $4.49 \mathrm{e}-10$ & $0.34 \mathrm{e}-30$ \\
\hline 7 & $\begin{array}{l}\text { Three-hump } \\
\text { Camel }\end{array}$ & -0.85 & -0.75 & -1 \\
\hline
\end{tabular}

Chicken Swarm Search algorithm has outperformed when compared with both Bat and Firefly algorithm. Chicken Swarm Search algorithm is very closer to global optima when compared with the both algorithms. From the table 1 it is understood that firefly algorithm is better for low dimensional functions. From the analysis of the given above table 1 it is experimented and proved that firefly algorithm performs much better when compared with bat algorithm for high dimensional functions and vice versa.

\section{B. Processing time}

Comparison between Bat, Firefly and Chicken Swarm Search algorithms based on processing time for these multi model functions could be given below using table 2 .

Table 2.processing time comparison in seconds for

\begin{tabular}{|l|l|l|l|l|}
\hline S.NO & FUNCTIONS & BAT & FFA & CS \\
\hline 1 & Ackley & 128.06 & 113.49 & 14.78 \\
\hline 2 & Egg holder & 159.37 & 119.03 & 28.86 \\
\hline 3 & Styblinski-Tang & 129.55 & 116.31 & 14.58 \\
\hline 4 & Fourth Schaffer & 127.18 & 113.44 & 11.58 \\
\hline 5 & The Mccormick & 12.36 & 10.28 & 0.91 \\
\hline 6 & Himmelblau's & 12.83 & 10.33 & 1.56 \\
\hline 7 & Three-hump Camel & 12.04 & 10.23 & 1.83 \\
\hline
\end{tabular}

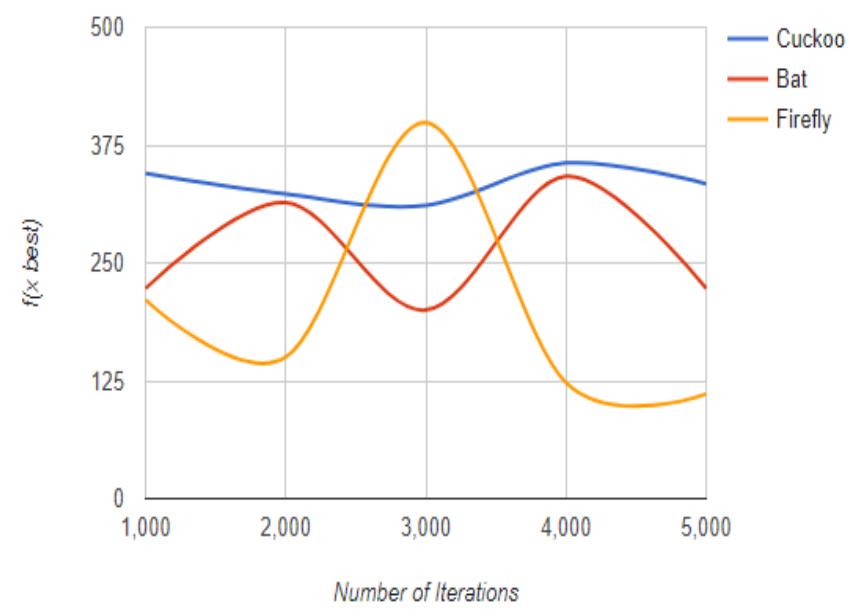

Fig 8. Convergence Plot For Ackley Function

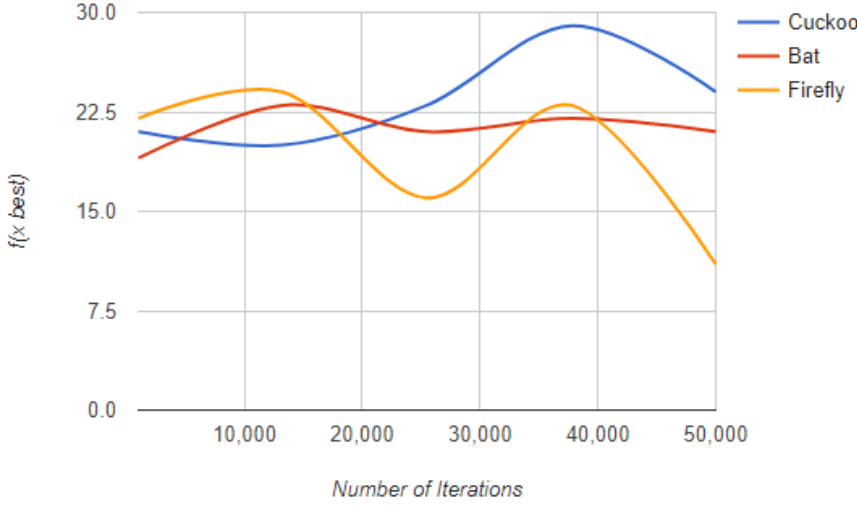

Fig 9. Convergence Plot Foregg Holder Function

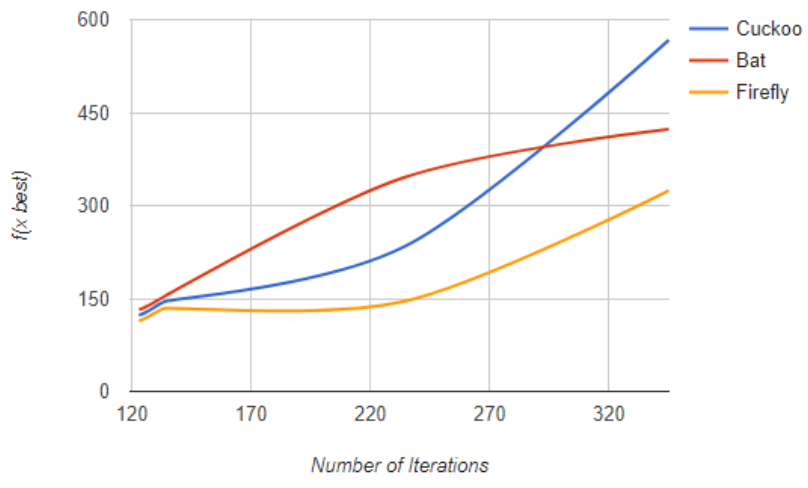

Fig 10. Convergence Plot For Styblinski-Tang Function

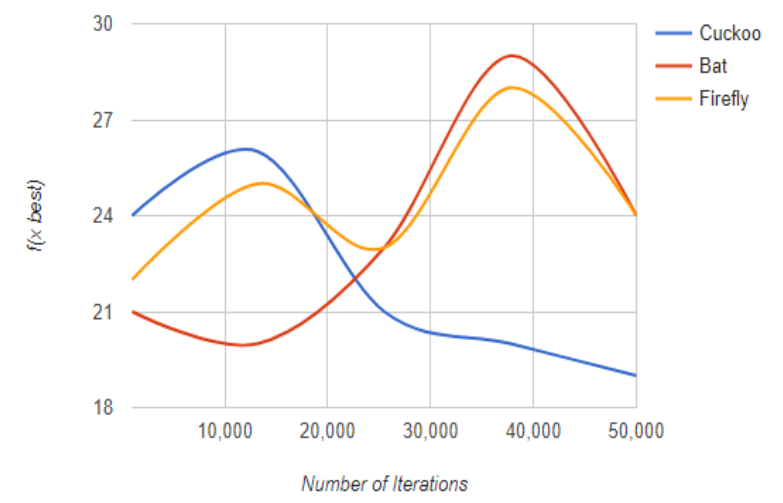

Fig 11. Convergence Plot For Fourth Schaffer Function 


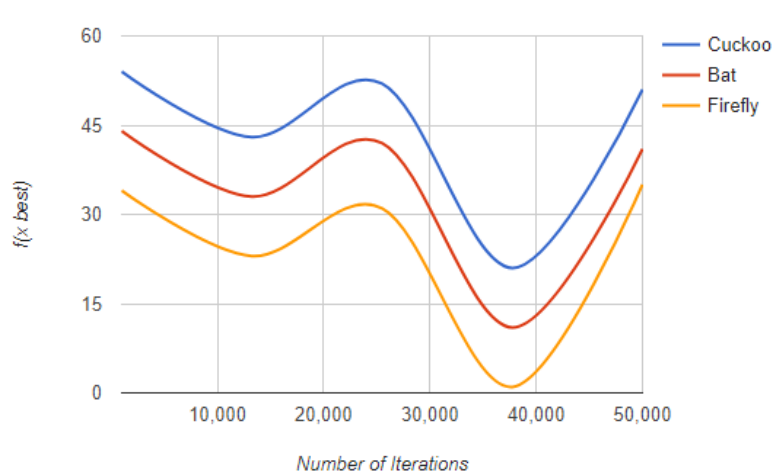

Fig 12.Convergence Plot For Mccormick Function

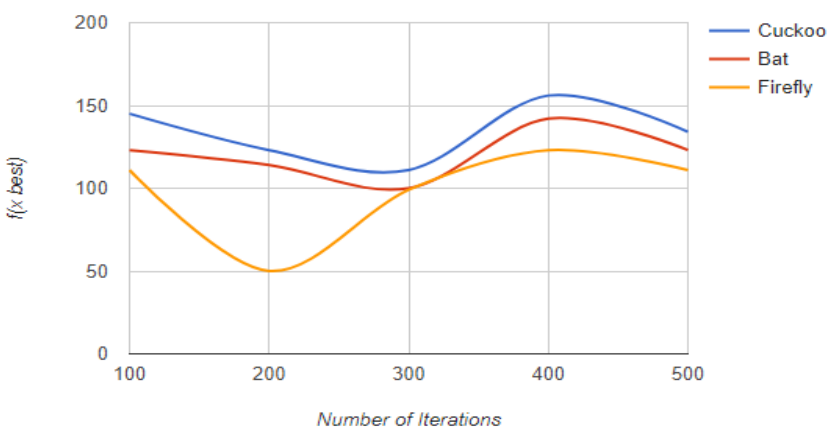

Fig 13. Convergence Plot For Himmelblau's Function

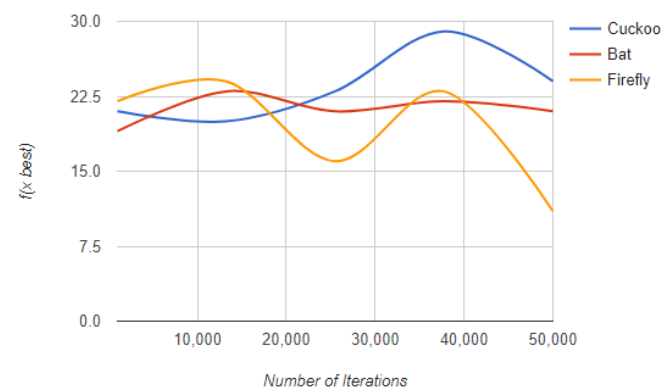

Figure 14. Convergence Plot For Three-Hump Camel Function

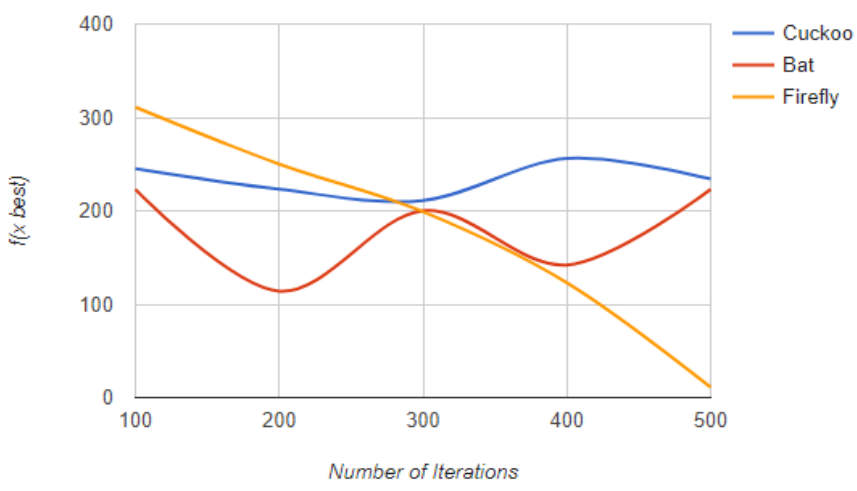

Fig 15. Convergence Plot For Ackley Function

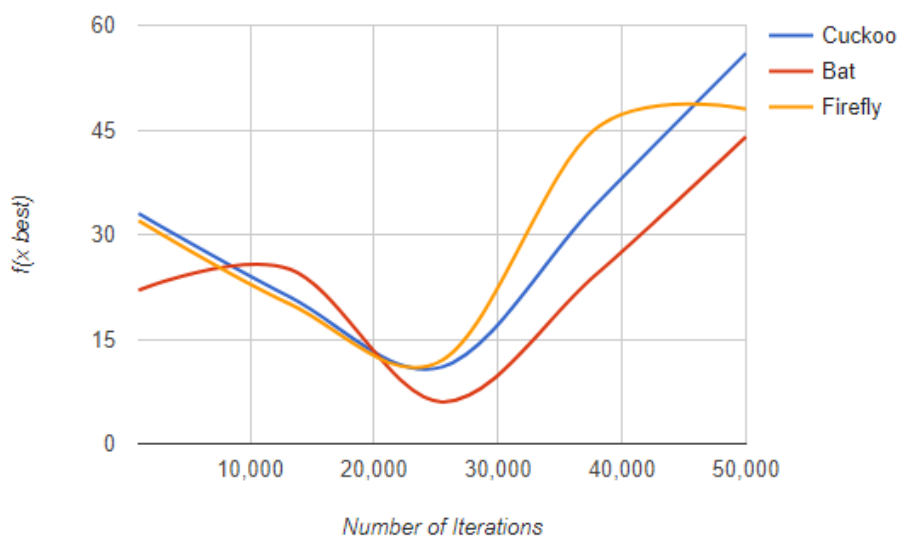

Fig 16. Convergence Plot Foregg Holder Function

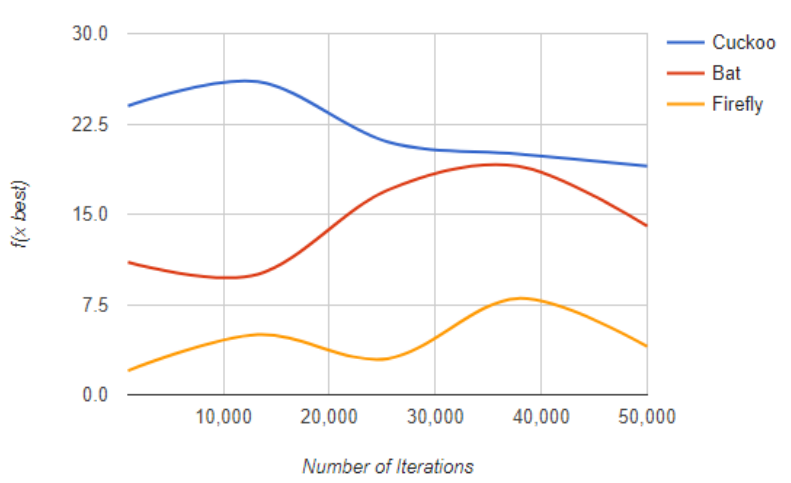

Fig 17. Convergence Plot For Styblinski-Tang Function

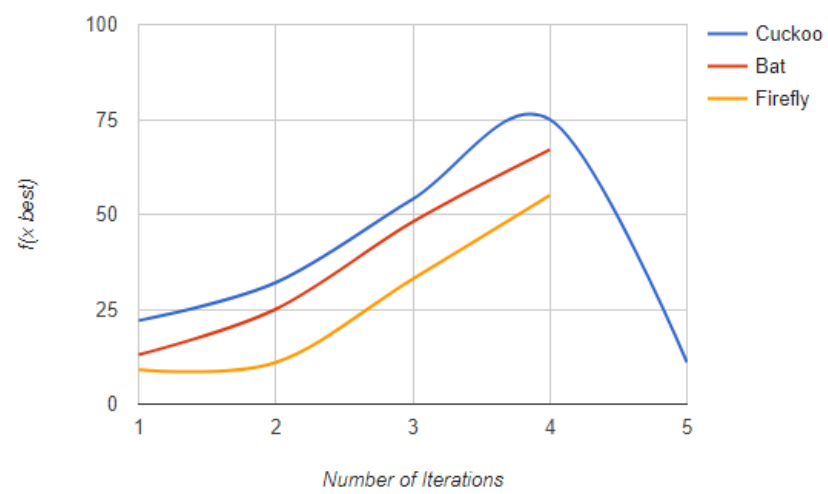

Fig 18. Convergence Plot For Fourth Schaffer Function

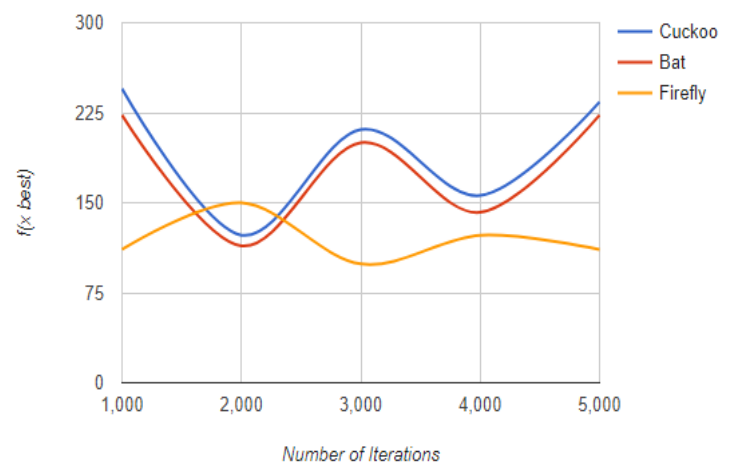

Fig 19.Convergence Plot For Mccormick Function 


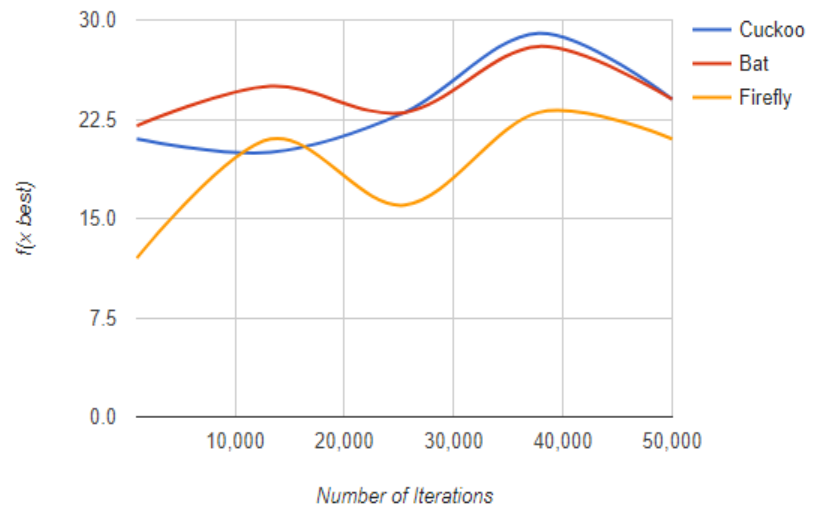

Fig 20. Convergence Plot For Himmelblau's Function Unimodal Test Functions

\section{Convergence}

Comparison between Bat, Firefly and Chicken Swarm Search algorithms based on convergence for these multi modal functions is given below using Figure 8-14.

\section{OPTIMIZATION FOR MULTIMODAL TEST FUNCTION}

\section{A. Fitness Calculation}

The performance of Bat algorithm, firefly algorithm and Chicken Swarm search algorithm are compared for various functions. Problems with low and high dimensional for both unimodal and multimodal are implemented for various test functions. The comparative is executed Using MATLAB and their computational procedures are executed. Simulations are used to compare and to do the experimental procedures for those above given three algorithms. Comparisons of optimization fitness for multimodal function are calculated using the given table below. When these three algorithms are compared with for multimodal test function Chicken Swarm perform better when compared with bat and firefly. Similarly, Chicken Swarm produce global optima when compared with other two algorithms.

\section{B. Processing time}

On the basis of processing time we compare all the three algorithms Firefly, Bat and Chicken Swarm search algorithm. Through single simulation the three algorithm Bat, firefly and Chicken Swarm search is compared. Single simulation also consumes time by fixed number of iteration to solve the problems. The time consumed by Chicken Swarm search algorithm is very low when compared with Firefly, Bat and Chicken Swarm search algorithm. Through Table 2 also it is proved such that Chicken Swarm earns much less time when compared with all the mentioned algorithms. As a result processing time for Chicken Swarm search executes in less time when compared with Firefly, Bat and Chicken Swarm search algorithms. It is proved such that the time taken by Chicken Swarm search algorithm is less when compared with the other two algorithms. It is also understood that from table 2 firefly compute less time when compared with bat algorithms.

\section{Convergence}

The Bat algorithm, Firefly algorithm, Chicken Swarm Search algorithm are compared for a particular number of iteration. Here it is optimized such that the Chicken Swarm estimated optimally well when compared with other two algorithms. Bat, Firefly and Chicken Swarm algorithm are plotted in Convergence graph in Figure 15-21.

\section{CONCLUSION}

Metaheuristic algorithm such as Bat algorithm, Firefly algorithm and Chicken Swarm search algorithm are compared using efficiency, time consumption and convergence graph. As a result it is achieved that Chicken Swarm search algorithm is optimized best when compared with the other two algorithms. Using efficiency, time consumption and convergence graph on unimodal and multimodal test functions; it is proved that Chicken Swarm is superior when compared with Bat algorithm, Firefly algorithm.

When comparison is made in between bat and firefly, Bat is much better when compared with Firefly algorithm for both low and high dimensional functions but in terms of time consumption. In multimodal optimization of both high and low dimensional functions Bat is much better when compared with Firefly algorithm. In unimodal optimization firefly algorithm is superior to bat algorithm for high dimensional function. Bat is fast of all when it is compared on the basis of convergence graph with the other two algorithms. As the dimension increases bat algorithm decreases with their fitness, though it decreases with its optimization fitness they perform better than Firefly for high dimensional multimodal function. Both unimodal and multimodal test function are equally capable of optimizing successfully they could effortlessly expand them to study multi objective optimization applications with a variety of constraints and even to NP-hard problems.

\section{REFERENCES}

1. X. S. Yang, "Nature-Inspired Metaheuristic Algorithms", Luniver Press, 2008

2. Sh. M. Farahani, A. A. Abshouri, B. Nasiri, and M. R. Meybodi, “A Gaussian Firefly Algorithm", International Journal of Machine Learning and Computing, Vol. 1, No. December 2011.

3. Sh. M. Farahani, A. A. Abshouri, B. Nasiri, and M. R. Meybodi, “A Gaussian Firefly Algorithm", International Journal of Machine Learning and Computing, Vol. 1, No. December 2011.

4. Xin-She Yang, Chaos-Enhanced Firefly Algorithm with Automatic Parameter Tuning, International Journal of Swarm Intelligence Research, December 2011

5. Gandomi A, Yang X-S, Alavi A, Talatahari S. Bat algorithm for constrained optimization tasks, Neural Computing and Applications, 22(2013) 1239-55

6. Yang X-S. A new metaheuristic bat-inspired algorithm, in: J González, D. Pelta, C. Cruz, G. Terrazas, N. Krasnogor (Eds.) Nature Inspired Cooperative Strategies for Optimization (NICSO 2010), Springer Berlin Heidelberg, 2010, pp. 65-74

7. G Thippa Reddy, N Khare FFBAT-optimized rule based fuzzy logic classifier for diabetes International Journal of Engineering Research in Africa 24, 137-152

8. GadekalluThippa Reddy NeeluKhare Hybrid Firefly-Bat Optimized Fuzzy Artificial Neural Network Based Classifier for Diabetes Diagnosis

9. International Journal of Intelligent Engineering and Systems, Vol.10, No.4, 2017

10. TR Gadekallu, N Khare -Chicken Swarm search optimized reduction and fuzzy logic classifier for heart disease and diabetes predictionInternational Journal of Fuzzy System Applications ISSN: 2156-177X|,2017

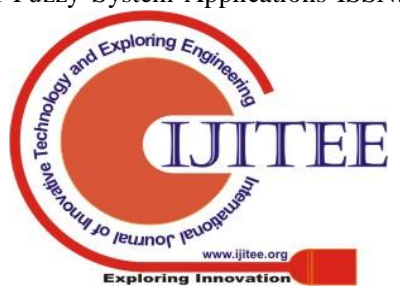


11. X.-S. Yang, S. Deb, "Chicken Swarm search via L'evy flights", in: Proc. Of World Congress on Nature \& Biologically Inspired Computing (NaBIC 2009), December 2009, India. IEEE Publications, USA, pp. 210-214 (2009).

12. GobindPreet Singh, Abhay Singh Comparative Study of Krill Herd, Firefly and Chicken Swarm Search Algorithms for Unimodal and Multimodal Optimization International Journal of Intelligent Systems and Applications in Engineering Advanced Technology and Science ISSN:2147-6799214, 2014, 2(3), 26-37

13. Yang, X.S., Deb, S.: Engineering Optimisation by Chicken Swarm Search. Int. J. Math. Model. Numer. Optim. 1(4), 330-343 (2010)

14. Manikandan P., Selvarajan S. (2014) Data Clustering Using Chicken Swarm Search Algorithm (CSA). In: Babu B. et al. (eds) Proceedings of the Second International Conference on Soft Computing for Problem Solving (SocProS 2012), December 28-30, 2012. Advances in Intelligent Systems and Computing, vol 236. Springer, New Delhi

15. Yang XS, Deb S (2009), Chicken Swarm search via levy flights. In: World congress on nature \& biologically inspired computing (NaBIC 2009). IEEE Publication, USA, pp 210-214

16. Xin-She Yang, Chaos-Enhanced Firefly Algorithm with Automatic Parameter Tuning, International Journal of Swarm Intelligence Research, December 2011

17. Sh. M. Farahani, A. A. Abshouri, B. Nasiri, and M. R. Meybodi, "A Gaussian Firefly Algorithm", International Journal of Machine Learning and Computing, Vol. 1, No. December 2011.

18. Vazquez, R. A., (2011). Training spiking neural models using Chicken Swarm search algorithm, 2011 IEEE Congress on Eovlutionary Computation (CEC'11), pp.679-686.

19. Ghulam Abbas, Jason Gu, Umar Farooq, Ali Raza, Muhammad UsmanAsad, M. E. El-Hawary, "Solution of an Economic Dispatch Problem Through Particle Swarm Optimization: A Detailed Survey Part II", Access IEEE, vol. 5, pp. 24426-24445, 2017, ISSN 2169. 3536.

20. SeyedJalaleddinMousavirad, HosseinEbrahimpour-Komleh, "Entropy based optimal multilevel thresholding using Chicken Swarm optimization algorithm", Innovations in Information Technology (IIT) 2015 11th International Conference on, pp. 302-307, 2015.

21. I.B. Saida K. Nadjet B. Omar "A new algorithm for data clustering based on Chicken Swarm search optimization" Genetic and Evolutionary Computing Springer pp. 55-64 2014.

\section{AUTHORS PROFILE}

K. Kalaivani, PSNA College of Engineering \& Technology, Department of Computer Science \& Engineering, Dindigul,Tamil Nadu,India.

Dr. N. UmaMaheswari, PSNA College of Engineering \& Technology, Department of Computer Science \& Engineering, Dindigul,Tamil Nadu, India. 